\title{
Dependence of AeroMACS Interference on Airport Radiation Pattern Characteristics
}

Jeffrey D. Wilson

Glenn Research Center, Cleveland, Ohio 


\section{NASA STI Program . . . in Profile}

Since its founding, NASA has been dedicated to the advancement of aeronautics and space science. The NASA Scientific and Technical Information (STI) program plays a key part in helping NASA maintain this important role.

The NASA STI Program operates under the auspices of the Agency Chief Information Officer. It collects, organizes, provides for archiving, and disseminates NASA's STI. The NASA STI program provides access to the NASA Aeronautics and Space Database and its public interface, the NASA Technical Reports Server, thus providing one of the largest collections of aeronautical and space science STI in the world. Results are published in both non-NASA channels and by NASA in the NASA STI Report Series, which includes the following report types:

- TECHNICAL PUBLICATION. Reports of completed research or a major significant phase of research that present the results of NASA programs and include extensive data or theoretical analysis. Includes compilations of significant scientific and technical data and information deemed to be of continuing reference value. NASA counterpart of peer-reviewed formal professional papers but has less stringent limitations on manuscript length and extent of graphic presentations.

- TECHNICAL MEMORANDUM. Scientific and technical findings that are preliminary or of specialized interest, e.g., quick release reports, working papers, and bibliographies that contain minimal annotation. Does not contain extensive analysis.

- CONTRACTOR REPORT. Scientific and technical findings by NASA-sponsored contractors and grantees.
- CONFERENCE PUBLICATION. Collected papers from scientific and technical conferences, symposia, seminars, or other meetings sponsored or cosponsored by NASA.

- SPECIAL PUBLICATION. Scientific, technical, or historical information from NASA programs, projects, and missions, often concerned with subjects having substantial public interest.

- TECHNICAL TRANSLATION. Englishlanguage translations of foreign scientific and technical material pertinent to NASA's mission.

Specialized services also include creating custom thesauri, building customized databases, organizing and publishing research results.

For more information about the NASA STI program, see the following:

- Access the NASA STI program home page at http://www.sti.nasa.gov

- E-mail your question to help@sti.nasa.gov

- Fax your question to the NASA STI Information Desk at 443-757-5803

- Phone the NASA STI Information Desk at 443-757-5802

- Write to: STI Information Desk NASA Center for AeroSpace Information 7115 Standard Drive Hanover, MD 21076-1320 


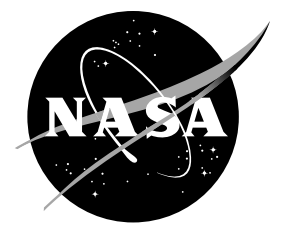

\section{Dependence of AeroMACS Interference on Airport Radiation Pattern Characteristics}

Jeffrey D. Wilson

Glenn Research Center, Cleveland, Ohio

Prepared for the

Integrated Communications, Navigation and Surveillance (ICNS) 2012 Conference

sponsored by the Institute of Electrical and Electronics Engineers

Herndon, Virginia, April 24-26, 2012

National Aeronautics and

Space Administration

Glenn Research Center

Cleveland, Ohio 44135 


\section{Acknowledgments}

The author would like to thank Chad Jeffrey Wilson for preparation of figures. The authors also gratefully acknowledge the Federal Aviation Administration's support of AeroMACS research at the NASA Glenn Research Center.

Trade names and trademarks are used in this report for identification only. Their usage does not constitute an official endorsement, either expressed or implied, by the National Aeronautics and Space Administration.

Level of Review: This material has been technically reviewed by technical management.

Available from

NASA Center for Aerospace Information 7115 Standard Drive

Hanover, MD 21076-1320
National Technical Information Service 5301 Shawnee Road Alexandria, VA 22312

Available electronically at http://www.sti.nasa.gov 


\title{
Dependence of AeroMACS Interference on Airport Radiation Pattern Characteristics
}

\author{
Jeffrey D. Wilson \\ National Aeronautics and Space Administration \\ Glenn Research Center \\ Cleveland, Ohio 44135
}

\begin{abstract}
AeroMACS (Aeronautical Mobile Airport Communications System), which is based upon the IEEE 802.16e mobile wireless standard, is expected to be implemented in the 5091 to $5150 \mathrm{MHz}$ frequency band. As this band is also occupied by Mobile Satellite Service (MSS) feeder uplinks, AeroMACS must be designed to avoid interference with this incumbent service. The aspects of AeroMACS operation that present potential interference are under analysis in order to enable the definition of standards that assure that such interference will be avoided. In this study, the cumulative interference power distribution at low earth orbit from AeroMACS transmitters at the 497 major airports in the contiguous United States was simulated with the Visualyse Professional software. The dependence of the interference power on the number of antenna beams per airport, gain patterns, and beam direction orientations was simulated. As a function of these parameters, the simulation results are presented in terms of the limitations on transmitter power required to maintain the cumulative interference power under the established threshold.
\end{abstract}

\subsection{Introduction}

Next generation air transportation systems will achieve high levels of efficiency and safety that will require major improvements in communications capacity and performance. In order to enable a safe and reliable airport surface communications network, development and test programs as well as the development of technical standards are being undertaken. This includes a test facility now in operation at the Cleveland Hopkins International Airport and the adjacent NASA Glenn Research Center (GRC) known as the Cleveland CNS Testbed. The testbed includes a prototype airport surface wireless communications network based on the IEEE 802.16e standard, known as Aeronautical Mobile Airport Communications System (AeroMACS) (Ref. 1).

The AeroMACS system is envisioned as a wireless network covering all areas of the airport surface and, if necessary, areas beyond where facilities and equipment might need to be located. The system would accommodate all mobile communications requirements including parked and taxiing aircraft, various types of ground vehicles, and personnel as well as connection to fixed assets related to airport safety requirements (such as surveillance and navigation aids, weather sensors, and communications stations).
AeroMACS is intended to operate in the 5091 to $5150 \mathrm{MHz}$ frequency region within C-band. It is essential that the AeroMACS service does not interfere with other users in this band. In particular, the allocation of the 5091 to $5150 \mathrm{MHz}$ band to the Earth-to-space fixed-satellite service (FSS), limited to feeder links of non-geostationary satellite systems in the mobile-satellite service (MSS), will restrict the power levels that will be allowed for AeroMACS networks. This investigation is focused on helping to establish practical limits on AeroMACS transmissions from airports so that the threshold of interference into MSS feeder links is not exceeded. This threshold interference power level at low Earth orbit (LEO) has been established at $-157.3 \mathrm{dBW}$ corresponding to a 2 percent increase of an MSS satellite receiver's noise temperature (Ref. 2).

Previously, the interference power distribution at LEO from AeroMACS transmitters at the 497 major airports in the contiguous United States was simulated with the Visualyse Professional software (Ref. 3). The results were shown to agree closely with those of a previous study by MITRECAASD (Ref. 4). Both omni-directional and sectoral antennas were modeled and 5 and $10 \mathrm{MHz}$ channels were considered with a center frequency of $5100 \mathrm{MHz}$.

In this study, the effect of the antenna gain profile on interference power is investigated and the accuracy of the model is improved by including a profile based on measured data. It is assumed that the channel bandwidth is $5 \mathrm{MHz}$ centered at $5100 \mathrm{MHz}$. The effect of the inhomogeneous distribution of airports will be examined by comparing with a case with the airports evenly distributed. Also the dependence of the interference power on the number of antenna beams and their directions at the airports will be simulated.

\subsection{Analysis}

The interference modeling was performed with Visualyse Professional Version 7 software from Transfinite Systems Limited (Ref. 5). Details of using this software were provided in (Ref. 6) with the modeling procedure summarized by the following seven steps:

1. Define antenna gain dependence on azimuthal and elevation angles.

2. Locate stations (transmitters and receivers).

3. Specify frequency and bandwidth of carriers.

4. Set up the propagation environment. 
5. Set up the links between stations.

6. Define victim and interfering links.

7. Specify desired output, submit run, and analyze results.

As in the MITRE-CAASD model (Ref. 4), the original NASA model (Ref. 3) assumed a worst case scenario with the antenna transmit gain pattern corresponding to peak side-lobe levels as specified by ITU (Ref. 7) with the transmitters on 100 percent of the time. A more recent European model (Ref. 8) differs in that it assumes average side-lobe levels for the antenna gain profile which is the distribution recommended by ITU (Ref. 7) for modeling the effects of a large number of antennas. With an assumption that the beamwidth is $120^{\circ}$, the antenna gains versus elevation and azimuth angles for the two models are shown in Figure 1 and Figure 2, respectively.

After the modeling was finished with the ITU-R peak and average side lobe antenna gain distributions, the measured gain distribution for the AeroMACS antennas became available from the manufacturer Alvarion (Ref. 9). A measurement-based gain profile was established by sampling

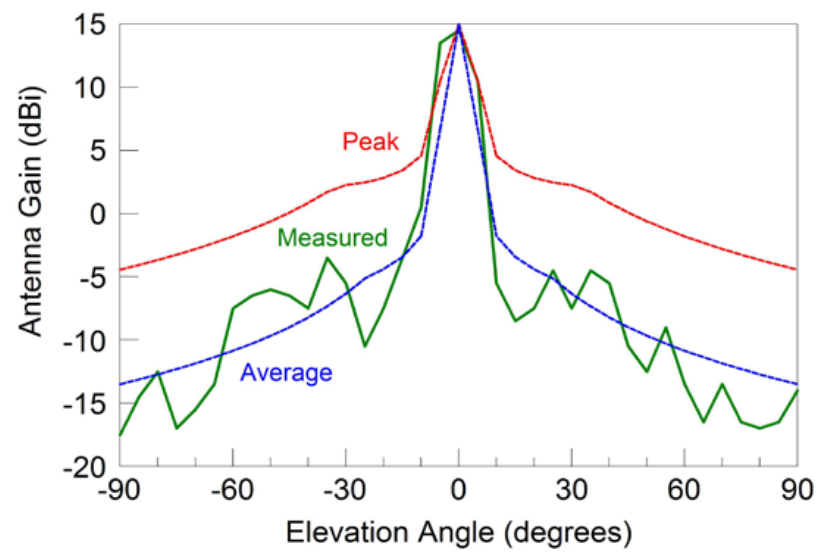

Figure 1.-Antenna gain versus elevation.

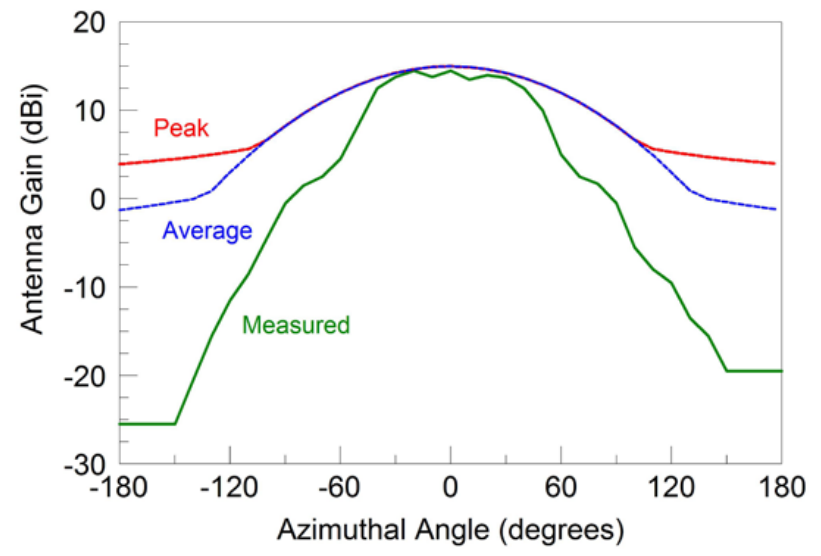

Figure 2.-Antenna gain versus azimuth. the $5100 \mathrm{MHz}$ data every $5^{\circ}$ and is also shown in Figure 1 and Figure 2. Figure 1 shows that the average side lobe model is a much better fit than the peak side lobe model. Figure 2 shows that there is considerable difference between the measured gains versus azimuthal angle from both of the models because the measured beamwidth is $80^{\circ}$ while the previous models assumed a $120^{\circ}$ beamwidth. The measured gain profile will be used in this investigation for improved accuracy.

\subsection{Results}

\subsection{Dependence of Interference Power Distribution Profile on Antenna Gain Profile}

The three antenna gain profiles (ITU-R Peak Side Lobe, ITU-R Average Side Lobe, and Measurement-Derived) were modeled for each beam and it was assumed that each of the 497 airports in the USA model utilized three beams. The beams were oriented $120^{\circ}$ from each other and the directions were randomized among the airports with representative randomizations shown in Figure 3. This orientation is referred to as Case 3.

Ten runs were performed with different randomizations for each profile and the average maximum interference powers were recorded. It was assumed that each beam transmits $-8.7 \mathrm{dBW}(135 \mathrm{~mW})$ of power. Figure 4, Figure 5, and Figure 6 show the resulting cumulative interference power for the peak side lobe, average side lobe, and measurement-derived gain profiles respectively for the Case 3 beam orientation. The scale for these and the following cumulative interference power figures is in dBW. The maximum interference power is -155.2 $\mathrm{dBW}$ for the peak side lobe case and $-156.9 \mathrm{dBW}$ for both the average side-lobe case and the measurement-derived case. Thus to be at or below the $-157.3 \mathrm{dBW}$ threshold everywhere, the transmitted power at each beam for the peak side lobe case needs to be decreased to $-8.7-(157.3-155.2)=-10.8 \mathrm{dBW}=$ $19.2 \mathrm{dBm}=\mathbf{8 3} \mathbf{~ m W}$. For both the average side lobe case and the measurement-derived case the transmitted power needs to be decreased to $-8.7-(157.3-156.9)=-9.1 \mathrm{dBW}=20.9 \mathrm{dBm}=$ $123 \mathrm{~mW}$. (Note that in all cases the transmitted power is calculated which is equal to the transmitter power minus the cable/line loss). Thus the previous NASA antenna gain model significantly underestimated the allowable transmitted power, while the European antenna gain model was a much better representation of the actual gain profile.

\subsection{Dependence of Interference Power Distribution Profile on Station Distribution}

In order to understand the effect of the inhomogeneous distribution of U.S. airports, a hypothetical case in which the 497 stations are evenly distributed over the contiguous United 


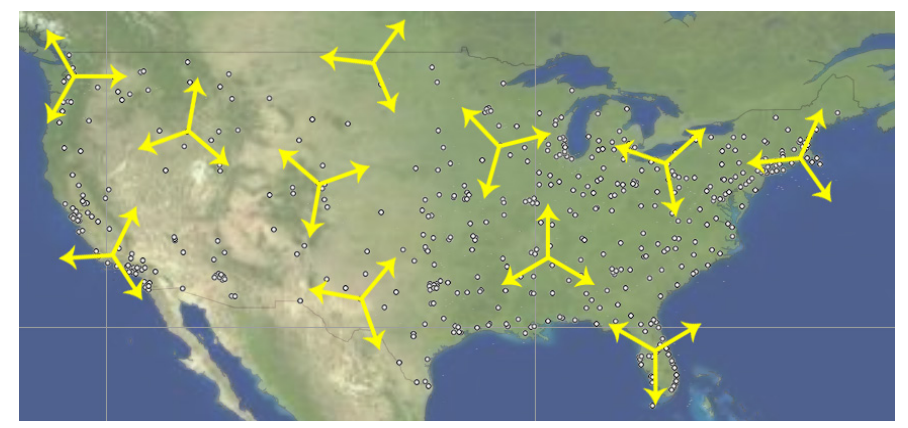

Figure 3.-Beam orientations for Case 3.

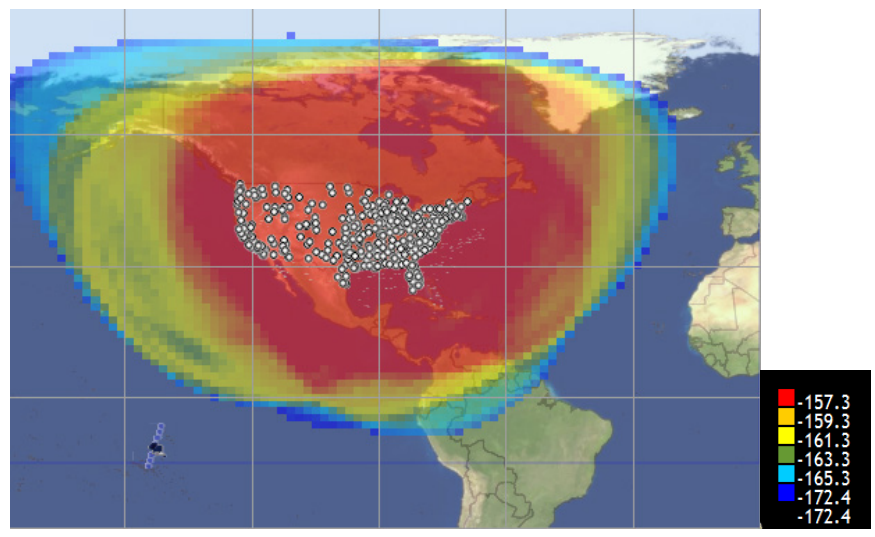

Figure 4.--Interference power from peak side lobe antenna gain.

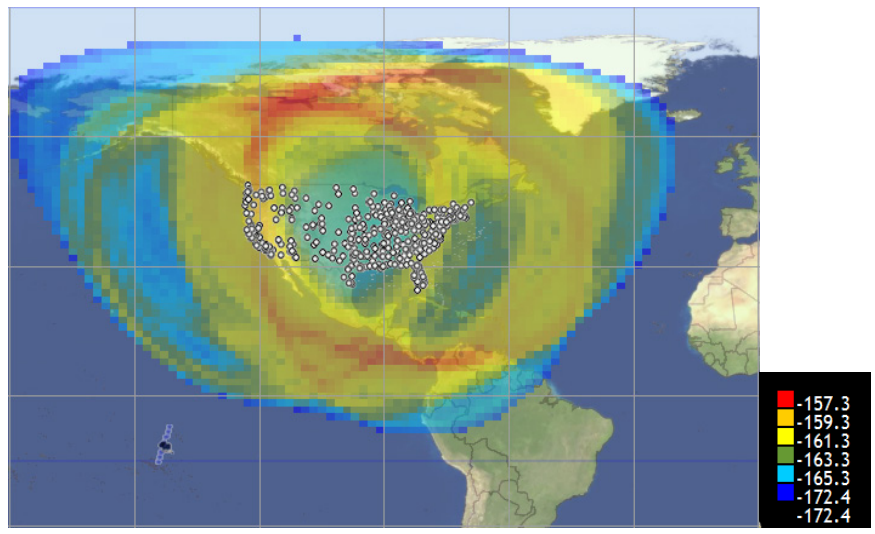

Figure 5.--Interference power from average side lobe antenna gain.

States was modeled as shown in Figure 7. The measurementderived antenna gain profile is used in this and all succeeding calculations. The maximum interference power is $-157.9 \mathrm{dBW}$. Thus to get below the $-157.3 \mathrm{dBW}$ threshold everywhere, the transmitted power for each beam needs to be decreased to $-8.7-(157.3-157.9)=-8.1 \mathrm{dBW}=21.9 \mathrm{dBm}=$ $155 \mathrm{~mW}$. This is $32 \mathrm{~mW}$ higher than with the real airport distribution. By spreading out the transmission sources, the cumulative power is less focused on the hot spot over northern Canada.

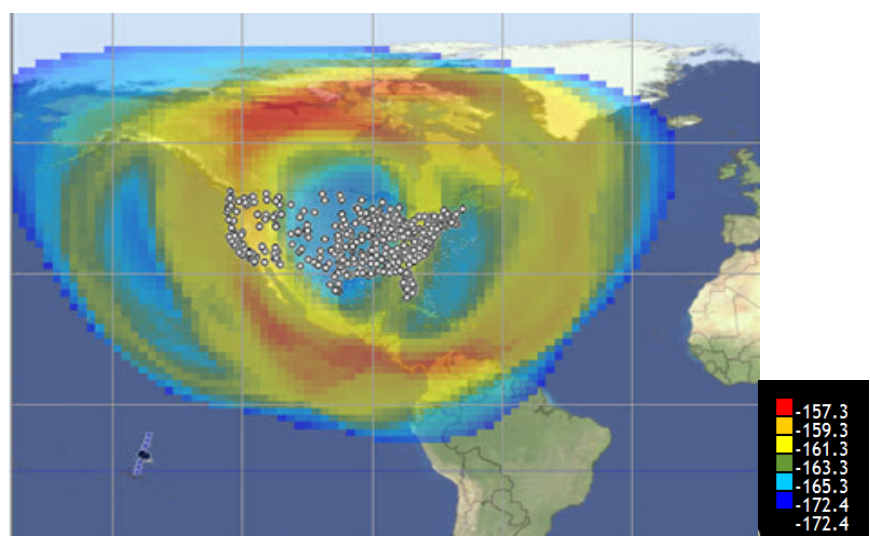

Figure 6.-Interference power from measurement-derived antenna gain.

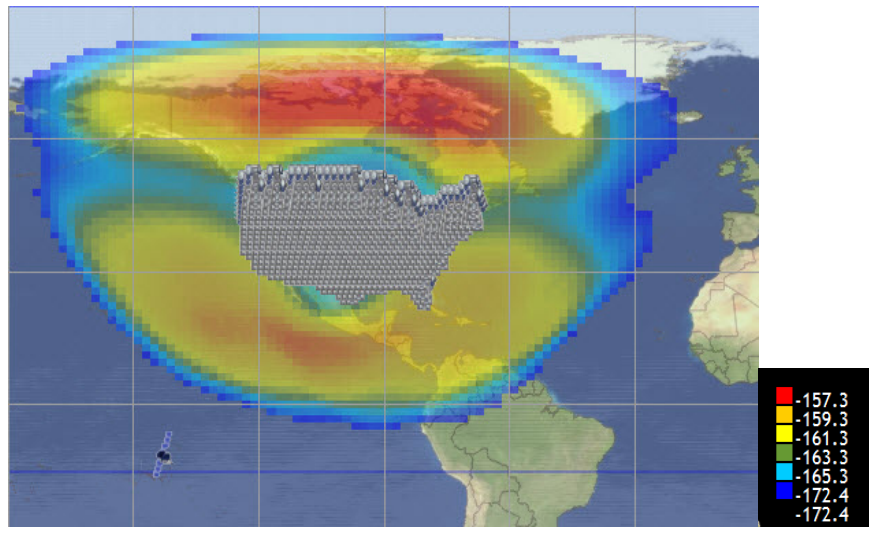

Figure 7.--Interference power for evenly distributed antennas.

\subsection{Dependence of Interference Power Distribution Profile on Beams per Airport}

To compare cumulative interference power with the case in Section 3.1 and Figure 4 where there were three beams at each of the 497 airports, results were also obtained with 1, 2, and 4 beams per airport. For each case, twenty runs were performed with different antenna orientation randomizations with each beam transmitting $-8.7 \mathrm{dBW}$ of power. For the two-beam case, two sub-cases were simulated: Case 2a with the beams separated by $180^{\circ}$ and Case $2 \mathrm{~b}$ with the beams separated by $120^{\circ}$. In the four beam case, the beams were separated by $90^{\circ}$. Representative randomized beam orientations for Cases 1, 2a, 2b, and 4 are shown in Figure 8 to Figure 11, respectively. (The beam orientations for Case 3 were shown in Figure 3). The cumulative interference power for sample runs of Cases 1 , 2a, and 4 beam are shown in Figure 12 to Figure 14, respectively.

For each run of the one-beam case, the maximum cumulative interference power at LEO altitude is obtained. For example in the run corresponding to Figure 11, this value is $-161.4 \mathrm{dBW}$ which occurs at latitude $67^{\circ} \mathrm{N}$ and longitude $105^{\circ} \mathrm{W}$ in northern Canada (all the runs for each of these cases result in a maximum cumulative interference power in 


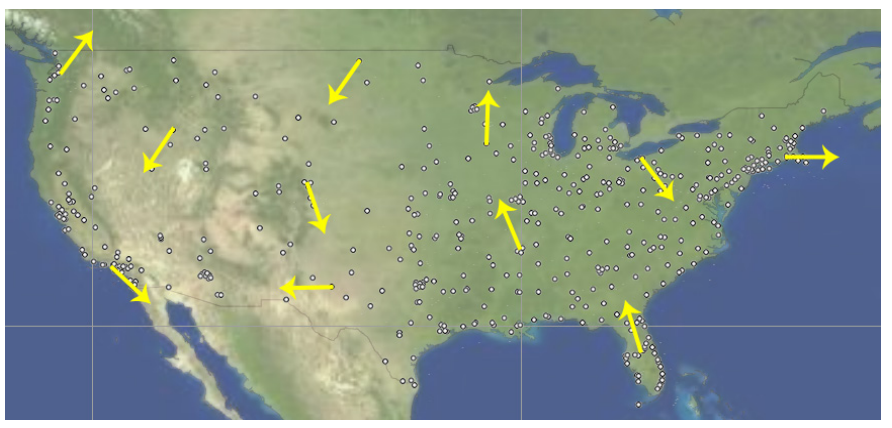

Figure 8.-Beam orientations for Case 1.

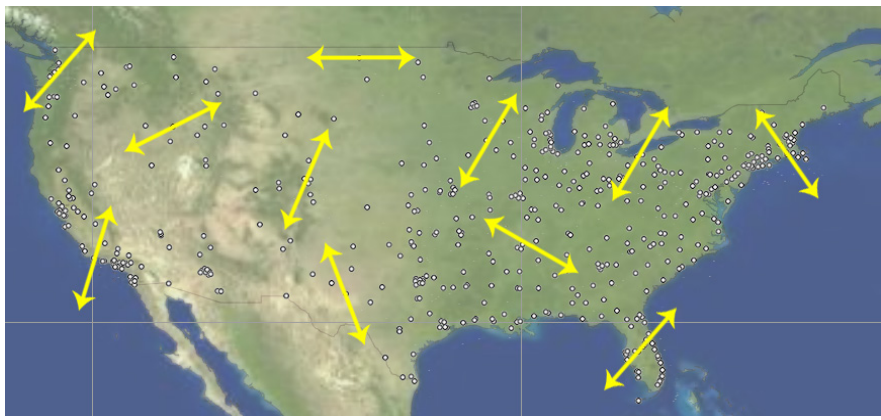

Figure 9.-Beam orientations for Case 2a.

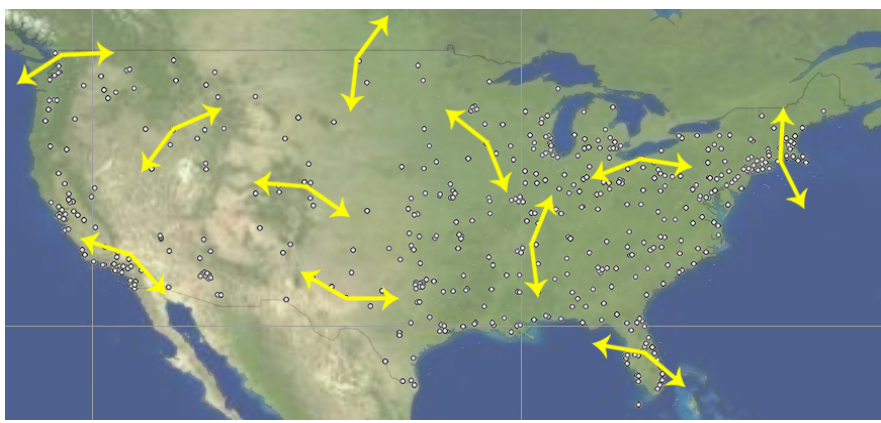

Figure 10.-Beam orientations for Case $2 b$.

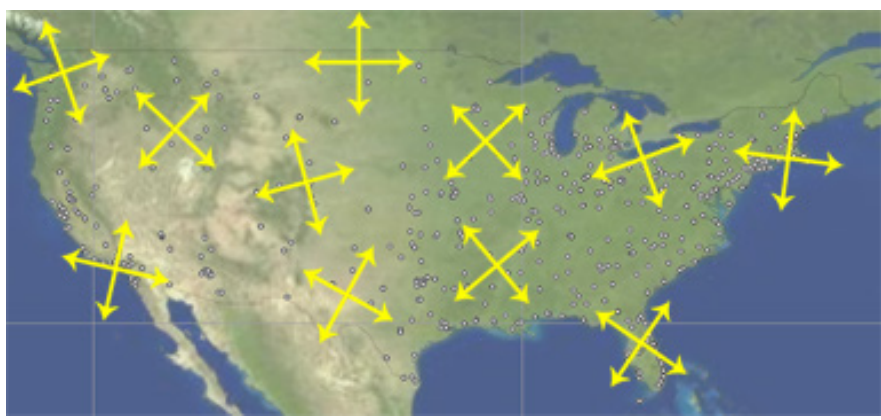

Figure 11.-Beam orientations for Case 4.

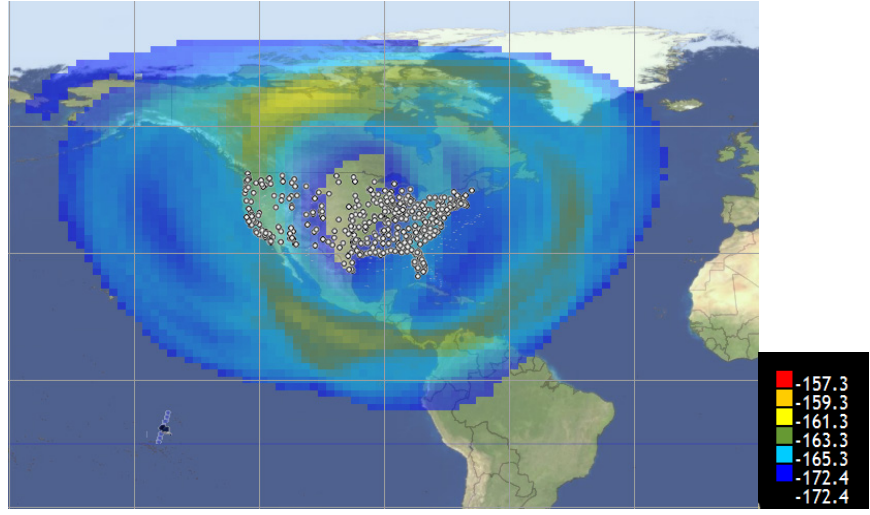

Figure 12.--Interference power for Case 1.

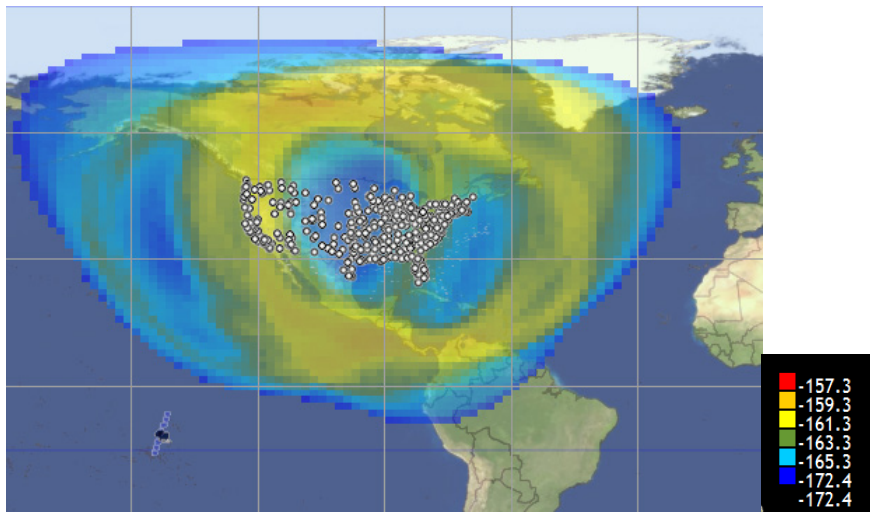

Figure 13.-Interference Power for Case 2a.

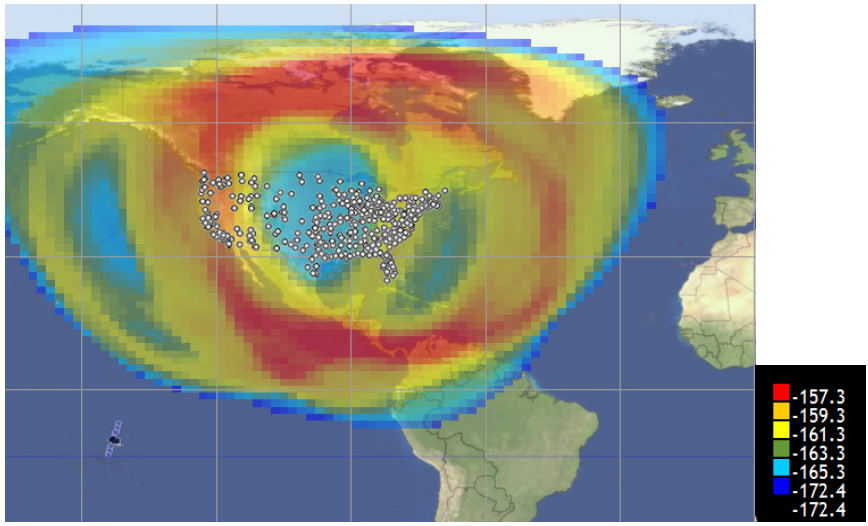

Figure 14.-Interference Power for Case 4 
northern Canada). The transmitted power that would result in the maximum cumulative interference power being equal to the threshold value of $-157.3 \mathrm{dBW}$ is calculated as $-8.7 \mathrm{dBW}$ $-157.3 \mathrm{dBW}-(-161.4 \mathrm{dBW})=-4.6 \mathrm{dBW}=343.7 \mathrm{~mW}$. This value along with those corresponding to the nineteen other one-beam runs is plotted as Case 1 in Figure 15. Similarly, the total power transmitted at each airport to provide a maximum cumulative interference power of $-157.3 \mathrm{dBW}$ is shown in Figure 15 for the two-beam $\left(180^{\circ}\right)$ case (Case 2a), two-beam $\left(120^{\circ}\right)$ case (Case $2 b$ ), three-beam case (Case 3), and fourbeam case (Case 4). It is seen that the variation between runs decreases as the number of beams per airport increases from one to four and the radiation pattern becomes more symmetrical.

The results of Figure 15 are summarized in Table I. For the twenty runs of each case, the table shows the average power and minimum power needed to reach the threshold cumulative interference power value of $-157.3 \mathrm{dBW}$. Also shown is the standard deviation $\sigma$, the average power value minus $2 \sigma$, and the average value minus $3 \sigma$. If we assume a normal distribution, these last two rows indicate that for a given random run, there is less than a 2.3 percent probability and less than a 0.2 percent probability that the value will fall below these respective values.

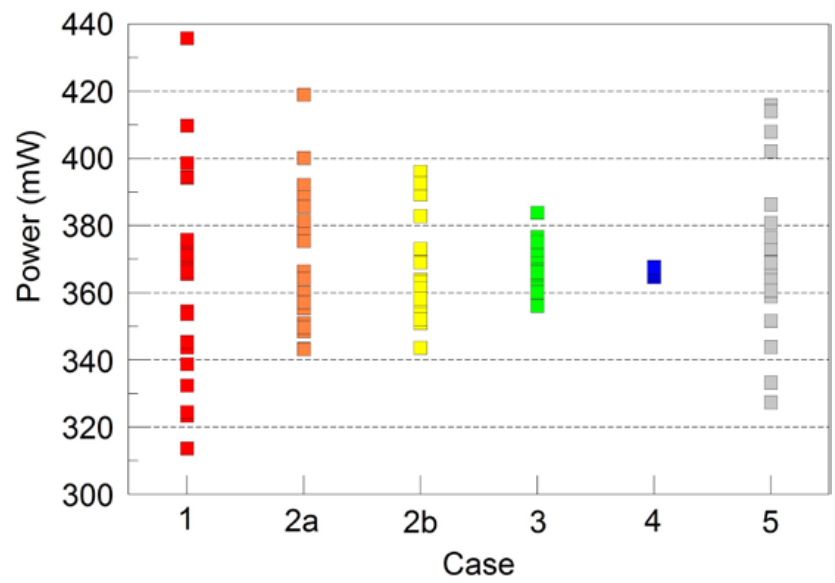

Figure 15.-Total power transmitted per airport to provide threshold interference power.

TABLE I.-STATISTICS FOR CASES 1 TO 6

\begin{tabular}{|c|c|c|c|c|c|c|c|}
\hline Case & 1 & $2 a$ & $2 \mathrm{~b}$ & 3 & 4 & 5 & 6 \\
\hline Description & $\begin{array}{c}1 \\
\text { beam }\end{array}$ & \begin{tabular}{|c|}
2 \\
beams \\
$180^{\circ}$
\end{tabular} & $\begin{array}{c}2 \\
\text { beams } \\
120^{\circ}\end{array}$ & $\begin{array}{c}3 \\
\text { beams } \\
120^{\circ}\end{array}$ & $\begin{array}{c}4 \\
\text { beams } \\
90^{\circ}\end{array}$ & $\begin{array}{c}1,2,3 \\
\text { and } 4 \\
\text { beams }\end{array}$ & $\begin{array}{c}2 \\
\text { beams } \\
\text { semi- } \\
\text { random }\end{array}$ \\
\hline Average power, mW & 364.2 & 369.7 & 366.3 & 365.9 & 366.3 & 371.5 & 176.2 \\
\hline Minimum power, mW & 313.6 & 343.2 & 343.6 & 356.1 & 364.7 & 327.3 & 151.3 \\
\hline$\sigma, \mathrm{mW}$ & 31.6 & 20.5 & 16.1 & 7.0 & 0.9 & 24.5 & 11.0 \\
\hline Average $-2 \sigma$, mW & 332.6 & 349.2 & 350.2 & 358.9 & 365.4 & 347.0 & 154.2 \\
\hline Average $-2 \sigma, \mathrm{mW}$ & 301.0 & 328.7 & 334.1 & 351.9 & 364.5 & 322.5 & 143.2 \\
\hline
\end{tabular}

\subsection{Dependence of Interference Power Distribution Profile on Airport Size}

Although there is a very large difference in size and power requirements among the 497 airports in this study, in the previous scenarios it was assumed that each transmitted an equivalent power. In order to provide a more realistic model, here the airports are divided into four size categories based on the number of passenger boardings in calendar year 2009 (Ref. 10). The 10 largest airports had more than 18 million passenger boardings and are designated 'very large' airports. Each very large airport is modeled with four beams. The next largest 18 airports had between 8 and 18 million boardings and are designated as 'large' airports and modeled with three beams. The next 33 are 'medium' airports with between 1.75 and 8 million boardings and modeled with two beams oriented $180^{\circ}$ apart. The remaining 436 airports with less than 1.75 million boardings are designated as 'small' airports and are modeled with one beam. (The codes for each of the very large, large, and medium airports are in the appendix.) This scenario is designated as Case 5 and representative orientations are shown in Figure 16.

The airport-averaged transmitted power is defined as the total power transmitted divided by the number of airports, 497 . For each of 20 runs with randomized orientations, the airportaveraged transmitted power that would result in the maximum cumulative interference power being equal to the threshold value of $-157.3 \mathrm{dBW}$ is shown in Figure 15 and the corresponding statistics are shown in Table I. The average number of beams at an airport is 1.199 , so for a power value in Figure 15 and Table I, the power transmitted by an individual airport is equal to the airport-averaged transmitted power multiplied by the number of beams and divided by 1.199. For example, in the run with the minimum airport-averaged transmitted power of $327.3 \mathrm{~mW}$, the 'very large' airports transmit $327.3 * 4 / 1.199=1091.9 \mathrm{~mW}$, the 'large' airports transmit 327.3*3/1.199 $=818.9 \mathrm{~mW}$, the 'medium' airports transmit 327.3*2/1.199 $=546.0 \mathrm{~mW}$, and the 'small' airports transmit 327.3/1.199 = $273.0 \mathrm{~mW}$.

From Figure 15, it is seen that the variation in the runs is less than in the one-beam scenario of Case 1, but more than the two-beam scenarios of Cases 2 and 3. This is to be

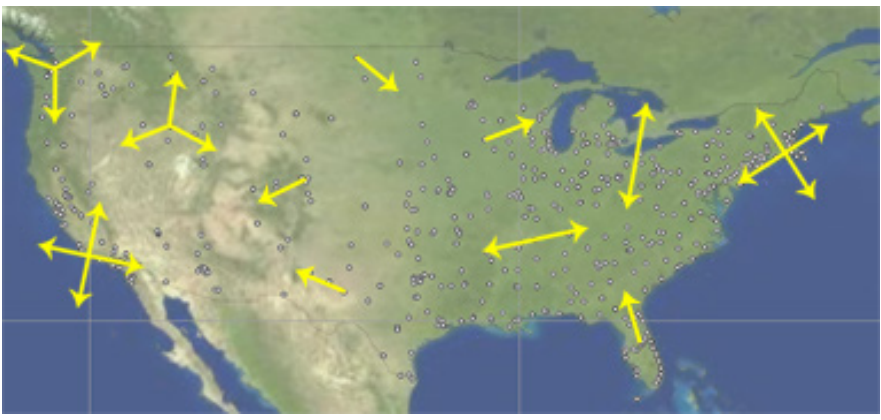

Figure 16.-Beam orientations for Case 5. 


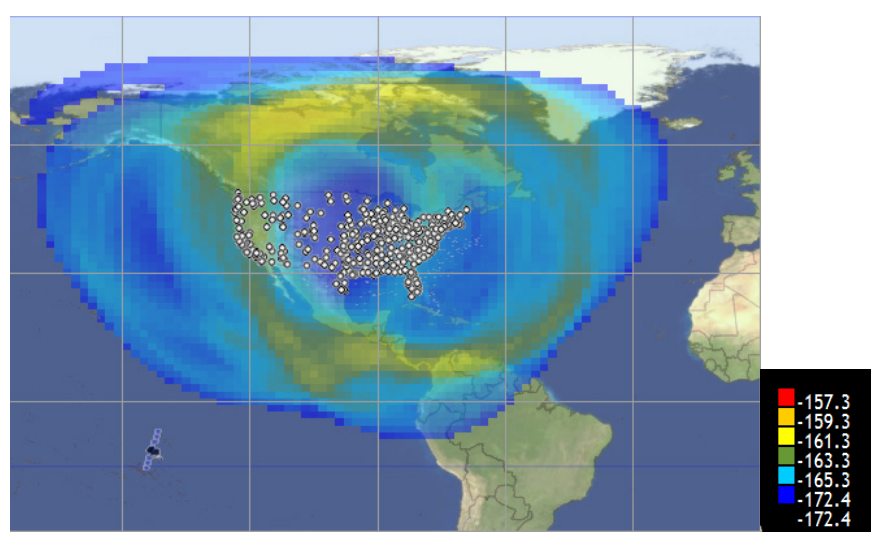

Figure 17.- Interference power for Case 5.

expected since the average number of beams per airport is 1.199. The cumulative interference power for a sample run with all beams transmitting $-8.7 \mathrm{dBW}$ is shown in Figure 17 .

\subsection{Dependence of Interference Power Distribution Profile on Semi-Randomized Beam Orientation}

The previous simulations have all assumed that the beam directions at each airport are randomly oriented. However since aircraft stability is enhanced when takeoff and landing are into the wind, runways are preferentially directed along the prevailing wind direction. In the continental United States this is generally from the southwest quadrant. In this section, an extreme case is modeled in which it is assumed that all the runways are oriented from the SW to the NE quadrant with half the base station antenna beams directed into the SW quadrant and half into the NE quadrant. In this case, referred to as Case 6, each airport has two beams separated by $180^{\circ}$ as in Case 2a. The directions within the SW-NE quadrants are randomly oriented with representative directions shown in Figure 18. This is an extreme case since not all runways are oriented in the SW-NE quadrants and not all base station beams will be directed along the runways; instead it is meant to serve as a bound for the lower limit of permissible transmit power per airport. It is expected that the future AeroMACS architecture will lie somewhere between the random orientations of the previous section and this semi-random orientation scenario.

Twenty runs were simulated, with a representative cumulative interference distribution shown in Figure 19. The 'hot spot' is now located over the Pacific Ocean west of Mexico instead of over northern Canada as in the random orientation cases in the previous section. The secondary 'hot spot' is over the northern Atlantic Ocean. The average transmitted power per airport to reach the threshold interference level at LEO is $176.2 \mathrm{~mW}, 47.7$ percent of the value for the corresponding random case, Case 2a. The worst run would allow only $151.3 \mathrm{~mW}$ to be transmitted per airport, 44.1 percent of that for the worst run of Case 2a. This should

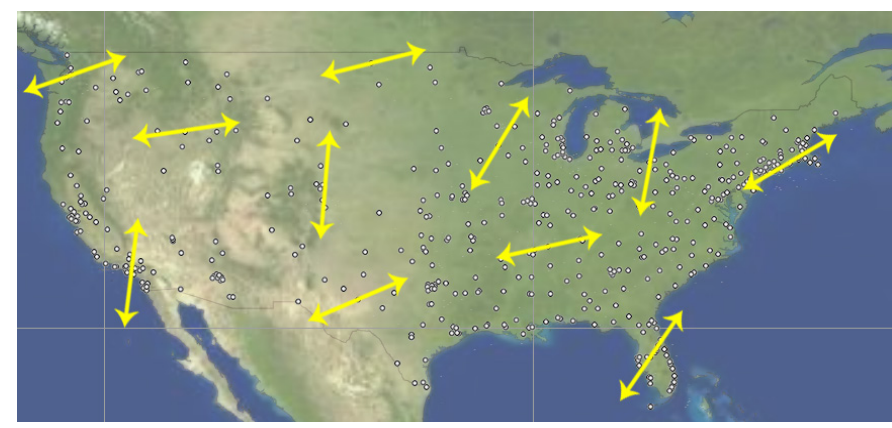

Figure 18.-Beam orientations for Case 6.

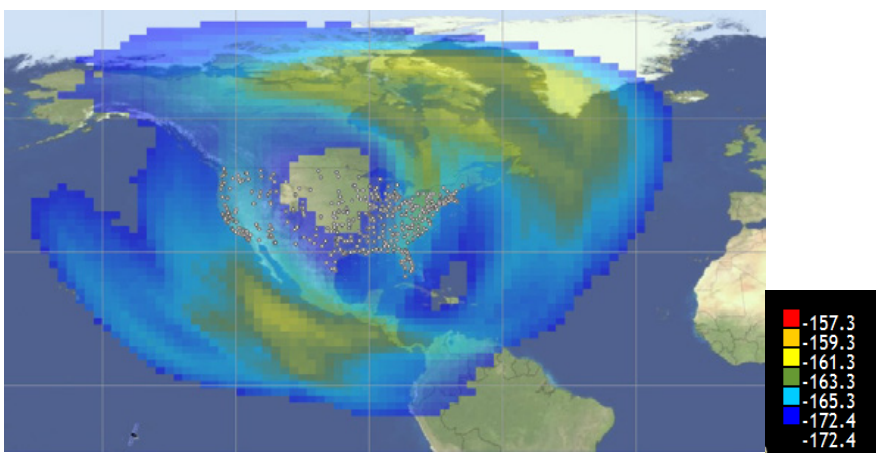

Figure 19._-Interference power for Case 6.

serve as a low level bound for allowable power to be transmitted per airport. In other words, the results suggest that there should be virtually no chance of causing interference at or above the threshold level if transmitted power is limited to less than $150 \mathrm{~mW}$ per airport. The limit per airport could be significantly higher, depending on the randomness of the antenna beam directions.

\subsection{Conclusions}

The effect of the AeroMACS base station antenna gain profile on cumulative interference power at the low Earth orbit (LEO) altitude was computationally investigated with the software package Visualyse Professional Version 7. It was assumed that base stations exists at each of the 497 major airports in the contiguous United States and each transmits over a single $5 \mathrm{MHz}$ channel centered at $5100 \mathrm{MHz}$ at all times. With the gain pattern based on experimental measurement, the number of beams at each base station was varied from one to four. For each case, twenty runs were simulated with different random orientations of the antenna beams and for each run, the transmitted power required to reach the threshold value was calculated. It was found that the average power that could be transmitted at each airport was about the same for each case and ranged from 364 to $370 \mathrm{~mW}$. However the variation among runs was large for asymmetric antenna gain patterns and small for symmetric patterns. The worst case scenario is for a single beam at each airport and allows up to $313.6 \mathrm{~mW}$ to be transmitted at each airport. 
In a more realistic model, the airports were divided into four size categories based on the number of passenger boardings in calendar year 2009. 'Very large', 'large', 'medium', and 'small' airports were modeled with four, three, two, and one beams respectively. Of the twenty runs with random antenna direction orientations, the worst case allowed an average airport transmission power of up to $327.3 \mathrm{~mW}$ to reach the threshold cumulative interference power at the Globalstar orbit. Assuming a normal distribution, the simulations indicated that there is less than a 0.2 percent probability that the interference threshold value will be exceeded if the average transmission power per airport is $322.5 \mathrm{~mW}$.

In all, twenty runs were simulated with each of six scenarios of base station antenna configurations with random beam direction orientations. For the 100 runs with equal power being transmitted at each airport, the worst case run allowed up to $313.6 \mathrm{~mW}$ to be transmitted at each airport before the cumulative threshold interference power is reached. In the scenario in which the larger airports are allowed to transmit more power, the worst case of 20 runs allowed up to an average of $327.3 \mathrm{~mW}$ per airport to be transmitted. This included the ten 'very large' airports transmitting up to $1091.9 \mathrm{~mW}$ each. It should be reiterated that the transmitted power is equal to the transmitter power minus the cable/line loss which is typically on the order of $1 \mathrm{~dB}$ and that the simulations assume the transmissions occur 100 percent of the time.

Because it is expected that the AeroMACS antenna beam directions will not be completely random, a semi-random case was modeled and examined. This extreme case assumed that half the base station antenna beams are randomly directed into the SW quadrant and half into the NE quadrant. This is a scenario that gives a much more concentrated cumulative interference power distribution than can be expected in future AeroMACS architectures and serves as a bound for the lower limit of permissible transmit power per airport. It is expected that the future AeroMACS architecture will lie somewhere between the random orientations of the random and semirandom scenarios. Twenty runs were submitted for this case and the worst run allows only $151.3 \mathrm{~mW}$ to be transmitted per airport before the threshold cumulative interference power limit is reached. This value is only 44.1 percent of the corresponding random case. The large difference indicates that the actual positioning of the AeroMACS antenna beam directions and their degree of randomness will have a large effect on the allowable transmitted power per airport. The results suggest that in a very bad scenario in which all antenna beams are directed into two quadrants, at least $150 \mathrm{~mW}$ in each channel can be transmitted at each airport while more optimal random beam orientations will allow more than $300 \mathrm{~mW}$ per channel. Since the 5091 to $5150 \mathrm{MHz}$ frequency band provides for up to eleven channels available to AeroMACS (Ref. 11), considerable capacity should be available without interfering with Mobile Satellite Service feeder uplinks.

The simulations do not include subscriber effects which are planned to be incorporated in a future study. 



\section{Appendix}

The 497 airports are designated as 'very large', 'large', 'medium', or 'small' according to the number of passenger boardings in calendar year 2009 (Ref. 5):

a. 10 very large airports with 2009 boardings $>18$ million, modeled with four beams: ATL, ORD, LAX, DFW, DEN, JFK, LAS, IAH, PHX, SFO

b. 18 large airports with 18 million $>2009$ boardings $>8$ million, modeled with three beams: CLT, EWR, MCO,

c. MIA, MSP, SEA, DTW, PHL, BOS, IAD, LGA, BWI, FLL, SLC, DCA, SAN, TPA, MDW d. 33 medium airports with 8 million > 2009 boardings $>1.75$ million, modeled with two beams: PDX, STL, CVG, MEM, MCI, CLE, OAK, SMF, RDU, BNA, SNA, SJC, HOU, AUS, PIT, MSY, MKE, SAT, IND, DAL, RSW, CMH, PBI, ABQ, JAX, BUF, BDL, ONT, BUR, PVD, OMA, RNO, TUS

e. 436 small airports with 2009 boardings $<1.75$ million, modeled with one beam: remaining towered airports in contiguous United States. 


\section{References}

1. Kerczewski, R. J., J. M. Budinger, T. J. Gilbert, 2008, Technology Assessment Results of the Eurocontrol/FAA Future Communications Study, IEEE Aerospace Conference.

2. Gheorghisor, I. L., Y.-S. Hoh, A. E. Leu, 2009, Analysis of ANLE Compatibility with MSS Feeder Links, MITRE-CAASD Report MTR090458.

3. Wilson, J. D., R. J. Kerczewski, 2011, Interference Analysis for an Aeronautical Mobile Airport Communications System, IEEE Aerospace Conference Proceedings, Paper 4.1503, Big Sky, Montana.

4. Hoh, Y.-S., I. L. Gheorghisor, A. E. Leu, 2005, Feasibility Analysis of 5091-5150 MHz Band Sharing by ANLE and MSS Feeder Links, MITRE-CAASD Report MP 05W0000083.

5. http://transfinite.com/content/downloadsvisualyse.html.

6. Wilson, J. D., 2011, Modeling C-Band Co-Channel Interference From AeroMACS Omni-Directional
Antennas to Mobile Satellite Service Feeder Uplinks, NASA/TM-2011-216938.

7. ITU-R, 2007, F-1336-2 Reference Radiation Patterns of Omni-directional, Sectoral and Other Antennas in Pointto-Multipoint Systems for Use in Sharing Studies in the Frequency Range from $1 \mathrm{GHz}$ to about $70 \mathrm{GHz}$.

8. J. E. Håkegård, 2011, Compatibility Study between AeroMACS and FSS, Proceedings of 2011 ICNS, Herndon, VA.

9. http://www.alvarion.com/index.php/products/breezemax/ breezemaxr-extreme-5000.

10. http://www.faa.gov/airports/planning_capacity/passenger _allcargo_stats/passenger/media/cy09_cs_enplanements. pdf.

11. Hall, E., J. Isaac, S. Henricksen, N. Zelkin, 2011, CBand Airport Surface Communications System Standards Development, Volume 1: Concepts of Use, Initial System Requirements, Architecture, and AeroMACS Design Considerations, NASA/CR - 2011216997/VOL1. 



\begin{tabular}{|c|c|c|c|c|c|}
\hline \multicolumn{5}{|c|}{ REPORT DOCUMENTATION PAGE } & $\begin{array}{l}\text { Form Approved } \\
\text { OMB No. 0704-0188 }\end{array}$ \\
\hline \multicolumn{6}{|c|}{ 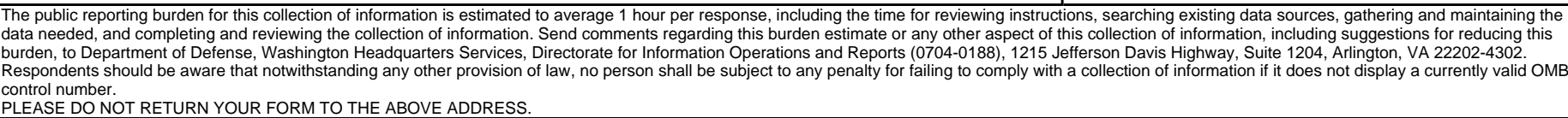 } \\
\hline \multicolumn{2}{|c|}{$\begin{array}{l}\text { 1. REPORT DATE (DD-MM-YYYY) } \\
01-08-2012\end{array}$} & \multicolumn{3}{|c|}{$\begin{array}{l}\text { 2. REPORT TYPE } \\
\text { Technical Memorandum }\end{array}$} & 3. DATES COVERED (From - To) \\
\hline \multirow{3}{*}{\multicolumn{5}{|c|}{$\begin{array}{l}\text { 4. TITLE AND SUBTITLE } \\
\text { Dependence of AeroMACS Interference on Airport Radiation Pattern Characteristics }\end{array}$}} & 5a. CONTRACT NUMBER \\
\hline & & & & & 5b. GRANT NUMBER \\
\hline & & & & & 5c. PROGRAM ELEMENT NUMBER \\
\hline \multirow{3}{*}{\multicolumn{5}{|c|}{$\begin{array}{l}\text { 6. AUTHOR(S) } \\
\text { Wilson, Jeffrey, D. }\end{array}$}} & 5d. PROJECT NUMBER \\
\hline & & & & & 5e. TASK NUMBER \\
\hline & & & & & $\begin{array}{l}\text { 5f. WORK UNIT NUMBER } \\
\text { WBS 031102.02.03.19.0677.12 }\end{array}$ \\
\hline \multicolumn{5}{|c|}{$\begin{array}{l}\text { 7. PERFORMING ORGANIZATION NAME(S) AND ADDRESS(ES) } \\
\text { National Aeronautics and Space Administration } \\
\text { John H. Glenn Research Center at Lewis Field } \\
\text { Cleveland, Ohio 44135-3191 }\end{array}$} & $\begin{array}{l}\text { 8. PERFORMING ORGANIZATION } \\
\text { REPORT NUMBER } \\
\text { E-18334 }\end{array}$ \\
\hline \multirow{2}{*}{\multicolumn{5}{|c|}{$\begin{array}{l}\text { 9. SPONSORING/MONITORING AGENCY NAME(S) AND ADDRESS(ES) } \\
\text { National Aeronautics and Space Administration } \\
\text { Washington, DC 20546-0001 }\end{array}$}} & $\begin{array}{l}\text { 10. SPONSORING/MONITOR'S } \\
\text { ACRONYM(S) } \\
\text { NASA }\end{array}$ \\
\hline & & & & & $\begin{array}{l}\text { 11. SPONSORING/MONITORING } \\
\text { REPORT NUMBER } \\
\text { NASA/TM-2012-217657 }\end{array}$ \\
\hline \multicolumn{6}{|c|}{$\begin{array}{l}\text { 12. DISTRIBUTIONIAVAILABILITY STATEMENT } \\
\text { Unclassified-Unlimited } \\
\text { Subject Category: } 04 \\
\text { Available electronically at http://www.sti.nasa.gov } \\
\text { This publication is available from the NASA Center for AeroSpace Information, 443-757-5802 }\end{array}$} \\
\hline \multicolumn{6}{|c|}{ 13. SUPPLEMENTARY NOTES } \\
\hline \multicolumn{6}{|c|}{$\begin{array}{l}\text { 14. ABSTRACT } \\
\text { AeroMACS (Aeronautical Mobile Airport Communications System), which is based upon the IEEE 802.16e mobile wireless standard, is } \\
\text { expected to be implemented in the } 5091 \text { to } 5150 \mathrm{MHz} \text { frequency band. As this band is also occupied by Mobile Satellite Service (MSS) } \\
\text { feeder uplinks, AeroMACS must be designed to avoid interference with this incumbent service. The aspects of AeroMACS operation that } \\
\text { present potential interference are under analysis in order to enable the definition of standards that assure that such interference will be } \\
\text { avoided. In this study, the cumulative interference power distribution at low earth orbit from AeroMACS transmitters at the } 497 \text { major } \\
\text { airports in the contiguous United States was simulated with the Visualyse Professional software. The dependence of the interference power } \\
\text { on the number of antenna beams per airport, gain patterns, and beam direction orientations was simulated. As a function of these parameters, } \\
\text { the simulation results are presented in terms of the limitations on transmitter power required to maintain the cumulative interference power } \\
\text { under the established threshold. }\end{array}$} \\
\hline \multicolumn{6}{|c|}{$\begin{array}{l}\text { 15. SUBJECT TERMS } \\
\text { Airports; Antenna gain; Antenna radiation patterns; Sidelobes }\end{array}$} \\
\hline \multicolumn{3}{|c|}{ 16. SECURITY CLASSIFICATION OF: } & $\begin{array}{l}\text { 17. LIMITATION OF } \\
\text { ABSTRACT }\end{array}$ & $\begin{array}{l}\text { 18. NUMBER } \\
\text { OF }\end{array}$ & $\begin{array}{l}\text { 19a. NAME OF RESPONSIBLE PERSON } \\
\text { STI Help Desk (email:help@sti.nasa.gov) }\end{array}$ \\
\hline $\begin{array}{l}\text { a. REPORT } \\
\text { U }\end{array}$ & $\begin{array}{l}\text { b. ABSTRACT } \\
\text { U }\end{array}$ & $\begin{array}{l}\text { c. THIS } \\
\text { PAGE } \\
\text { U }\end{array}$ & UU & $\begin{array}{l}\text { PAGES } \\
18\end{array}$ & $\begin{array}{l}\text { 19b. TELEPHONE NUMBER (include area code) } \\
443-757-5802\end{array}$ \\
\hline
\end{tabular}



